\title{
C4orf7 contributes to ovarian cancer metastasis by promoting cancer cell migration and invasion
}

\author{
CHANGYU WANG* ${ }^{*}$ LI ZHOU*, SHUANG LI, JUNCHENG WEI, WEI WANG, TING ZHOU, SHUJIE LIAO, \\ DANHUI WENG, DONGRUI DENG, YANJIE WENG, SHIXUAN WANG and DING MA \\ Cancer Biology Research Center, Tongji Hospital, Tongji Medical College, \\ Huazhong University of Science and Technology, Wuhan, Hubei 430030, P.R. China
}

Received December 2, 2009; Accepted March 2, 2010

DOI: $10.3892 /$ or_00000939

\begin{abstract}
Gene C4orf7, renamed FDC-SP (follicular dendritic cell secreted protein, FDC-SP) was first isolated from human tonsils. Up to the present, the function of this gene was still poorly understood. In this study, we report the expression of gene C4orf7 in a panel of tumor types. The percentages of C4orf7 positive expression in the tumor patients with metastases were notably higher than those in the cancer without metastases. On the contrary, the expression was hardly noted in normal tissues and corresponding benign lesions. In vitro, the up-regulation of C4orf7 in ovarian cancer cell lines was associated with enhanced motility and invasiveness. Furthermore, the overexpression of C4orf7 resulted in Akt ser473 phosphorylation and decreased E-cadherin expression. The role of C4orf7 in ovarian cancer cell morphology, motility and invasion was demonstrated.
\end{abstract}

\section{Introduction}

Ovarian cancer has the highest mortality among the gynecologic malignancies (1). The 5-year survival rate varies from $90 \%$ for patients diagnosed at the early stage of the disease to $<30 \%$ for the late-stage patients $(2,3)$. Therefore, successful outcome relies heavily on early diagnosis (4). Invasion and metastasis are characteristics of malignancy. It is the process by which cancer cells disseminate from the primary

Correspondence to: Dr Ding Ma or Dr Shixuan Wang, Cancer Biology Research Center, Tongji Hospital, Tongji Medical College, Huazhong University of Science and Technology, Wuhan, Hubei 430030, P.R. China

E-mail:dma@tjh.tjmu.edu.cn; sxwang@tjh.tjmu.edu.cn

*Contributed equally

Abbreviations: EOC, epithelia ovarian cancer; $\mathrm{p}-\mathrm{AKT}^{\mathrm{Ser} 473}$, phosphorylated Akt at serine 473

Key words: C4orf7, ovarian cancer, invasion, Akt neoplasm and invade surrounding tissue and survive in distant organs. Metastasis is the primary cause of morbidity and mortality for cancer patients (5).

Lymphatic metastases frequently occur in carcinomas and are seen as a key factor for staging and an important prognostic indicator of poor outcome $(6,7)$. However, the mechanism of interaction between tumor cells and endothelial cells is still poorly understood. In a previous study, a novel method was developed for the analysis of global gene expression profiles in lymphatic endothelial cells (LECs), which successfully combined laser capture microdissection (LCM) with microarray (8). The result of the microarray indicated a significant difference in the expression level of gene C4orf7 (chromosome 4 open reading frame 7) in LECs between ovarian cancer and normal tissues.

Gene C4orf7, renamed FDC-SP (follicular dendritic cell secreted protein, FDC-SP) was first isolated from human tonsils in 2002 (9). Previous studies on this gene were focused on its mediator function of B lymphoma cells. Al-Alwan et al (10) reported that $\mathrm{C} 4$ orf7 was a unique secreted peptide with a distinctive expression pattern within the immune system and had the ability to specifically bind to activated B cells. Up to the present, the function of this gene was poorly understood.

Gene C4orf7 has no significant similarity to known genes in GenBank. More than 30 sequences matching its sequence are largely derived from fetal tissue or tumor cell libraries (9), indicating that it may play an important role in embryonic development or tumorigenesis. However, whether is it related to tumor invasion and metastasis, is not known. Why does it have a high expression level in tumor tissues while low in normal tissues?

In order to explain the above results, the expression pattern of C4orf7 was investigated in several types of carcinoma specimens, including breast carcinoma, epithelial ovarian cancer, endometrial carcinoma as well as prostate cancer. In this study, the co-expression of C4orf7 in tumor cells and endothelial cells was first demonstrated, especially in epithelial ovarian cancer (in which it has the highest positive rate). It was also expressed in FLT-4 positive LECs of tumor tissues. Our results indicated that the overexpression of C4orf7 was related to tumorigenesis and cancer metastasis.

To further investigate the role of $\mathrm{C} 4$ orf 7 on malignant behaviors of ovarian cancer cells, the eukaryotic expression 
vector for C4orf7 and C4orf7 antisense RNA were constructed and then transfected into ovarian cancer cell lines A2780 and Skov3, respectively. Our research showed that the silence of C4orf7 resulted in decreased cell motility and invasiveness in Skov3 cell clones while enhanced expression of A2780 clones tend to have more migration and invasive capability. Blocking of C4orf7 also induced reorganization of cytoskeleton, in the form of disruption of the F-actin. Furthermore, we verified that enhanced C4orf7 expression caused down-regulation of E-cadherin by increasing phosphorylation of Akt ser473.

\section{Materials and methods}

Tissue samples and cell lines. Formalin-fixed, paraffinembedded tissue specimens diagnosed from January 2005 to December 2008, including breast duct carcinoma (163 cases), epithelial ovarian carcinoma (EOC, 80 cases), borderline ovarian tumor (15 cases) endometrial carcinoma (35 cases) and prostate cancer (30 cases), as well as their corresponding normal tissues (15 cases, respectively), were selected from Tongji Hospital, Huazhong University of Science and Technology, Wuhan, China.

SKOV3 cell line was obtained from the American Type Tissue Collection (ATCC). A2780 cell line was purchased from European collection of cell cultures (EATCC). The cell lines were maintained in Dulbecco's modified Eagle's medium (DMEM) containing 10\% fetal bovine serum in a humidified atmosphere supplemented with $5 \% \mathrm{CO}_{2}$ at $37^{\circ} \mathrm{C}$.

Immunohistochemistry. Paraffin sections (5 $\mu \mathrm{m}$ thickness) were dewaxed in xylene and hydrated in a serial of dilutions of alcohol. For antigen retrieval, we use $10 \mathrm{mM}$ citrate buffer, pH 6.0 as antigen unmasking solution. Sections were microwaved for $15 \mathrm{~min}$ and left to cool down at RT. Then endogenous peroxidase was quenched in $0.3 \%$ hydrogen peroxide in methanol for $15 \mathrm{~min}$. Primary antibody included rabbit anti-human C4orf7 IgG (1:100 dilution, Boster Biological Technology) and VEGFR3 (1:150 dilution Santa Cruz Biotechnology). Then tissue sections were stained using standard protocols according to SP method (Shanghai Changdao Biotech Co., Ltd.). The negative controls were incubated with non-immune mouse immunoglobuling ( $\mathrm{IgG}$ ) in place of the primary antibody. The immunostained slides were examined under a light microscope and scored independently by two experienced pathologists in the pathological department of Tongji Hospital, the score criteria was conducted as previously described (11).

RT-PCR and real-time-PCR. For RT-PCR, total RNA was isolated from cell lines with TRIzol reagent (Invitrogen, Life Technologies, CA) and cDNA was synthesized from $5 \mu \mathrm{g}$ of total RNA using Superscript reverse transcriptase (Life Technologies, Inc.) as per the manufacturer's instructions. The following primers were used: C4orf7: sense, 5'-CGG GATCCATGAAGAAAGTTCTCCTCCTGA-3'; antisense, 5'-CGGAATTCCTTTTCGCTAGGAAGGGGAG-3'. GAPDH: sense, 5'-TGAAGGTCGGAGTCAACGGATT TGGT-3'; antisense, 5'-CATGTGGGCCATGAGGTCAC CAC-3'.
PCR consisted of 30 cycles was carried out at the following conditions: $95^{\circ} \mathrm{C}$ for $5 \mathrm{~min}, 30$ cycles of $94^{\circ} \mathrm{C}$ for $30 \mathrm{sec}$, annealing at $48^{\circ} \mathrm{C}$ for $45 \mathrm{sec}$ and elongation at $72^{\circ} \mathrm{C}$ for $45 \mathrm{sec}$. PCR products were analyzed on $1.5 \%$ agarose gel and visualized under UV light after staining with ethidium bromide.

For real-time-PCR, RNA was extracted by using TRIzol and transcribed into cDNA with high-capacity cDNA kit (QianGene). The relative levels of mRNA gene expression were calculated with the $2^{-\Delta \Delta C t}$ method. The primers were as follows: C4orf7, 5'-GCTACAATATTCCAGGGCCAGTC-3'; 5'-CAACAGCCACTGCCAAGATG-3'.

Plasmid construction and cell transfection. C4orf7 cDNA was obtained by RT-PCR using SKOV3 RNA as a template. The amplified sequence was inserted into the pEGFPN1 and pEGFPC1 plasmid vector in an antisense orientation. The pEGFPC1 and pEGFPN1 plasmid expressing green fluorescent protein (GFP) were selected (BD Biosciences Clontech, Palo Alto, CA). All plasmid constructs were verified by nucleotide sequence analysis. The empty vector, pEGFPN1C4orf7 plasmid (sense) and pEGFPC1-C4orf7 plasmid (antisense) were transfected into A2780 and SKOV3 cells with lipofectamine 2000 reagent (Invitrogen, Life Technologies) according to the manufacturer's protocol, respectively. EGFP protein expression was detected under fluorescent microscopy. Colones were selected and maintained in the presence of $400 \mu \mathrm{g} / \mathrm{ml} \mathrm{G} 418$. Real-time PCR was applied to analyze C4orf7 mRNA expression level.

Western blot analysis. Total cell lysates were prepared in a lysis buffer $(20 \mathrm{mM}$ Tris $\mathrm{pH} 7.5,150 \mathrm{mM} \mathrm{NaCl}, 1 \mathrm{mM}$ EDTA, $1 \mathrm{mM}$ EGTA, 1\% Triton X-100, $2.5 \mathrm{mM}$ sodium pyrophosphate, $1 \mathrm{mM}$ h-glycerolphosphate, $1 \mathrm{mM}$ sodium orthovanadate, $0.5 \mathrm{mM}$ phenylmethylsulfonyl fluoride, and $1 \mathrm{mg} / \mathrm{ml}$ leupeptin) with a cocktail of protease inhibitors. Lysates were cleared by centrifugation and mixed with SDS sample buffer containing Tris pH 6.8, 2\% SDS, glycerol and B-mercaptoethanol. The protein concentration was estimated and $50 \mu \mathrm{g}$ of total protein extracts were separated by SDSPAGE, and transferred to NC membranes. The membranes were subsequently blocked with $5 \%$ skim-milk in TBS-T (50 mM Tris pH 7.5, $150 \mathrm{mM} \mathrm{NaCl}$, and $0.1 \%$ Tween-20) for $1 \mathrm{~h}$ at RT, and incubated overnight at $4^{\circ} \mathrm{C}$ with the primary antibodies including Akt (R\&D systems), phosphoAkt (R\&D systems) and $\beta$-actin (Santa Cruz Biotechnology). Actin was used as internal positive control. Protein was visualized by using enhanced chemiluminescence system (ECL, Pierce Biotechnology, USA).

Wound healing assay. Cells grown in 12-well plates as confluent monolayer were scratched with a $20-\mu 1$ pipette tip to create the wound. Then cells were washed with PBS three times to remove the debris, and serum-free culture media were added to the plates. Phase contrast images of the wound were taken at three random locations immediately after wounding and then at the same location after $24 \mathrm{~h}$ to examine wound closure by migrating cells. The distance of wound closure (compared with control at $0 \mathrm{~h}$ ) was measured in three independent wound sites per sample. The relative 

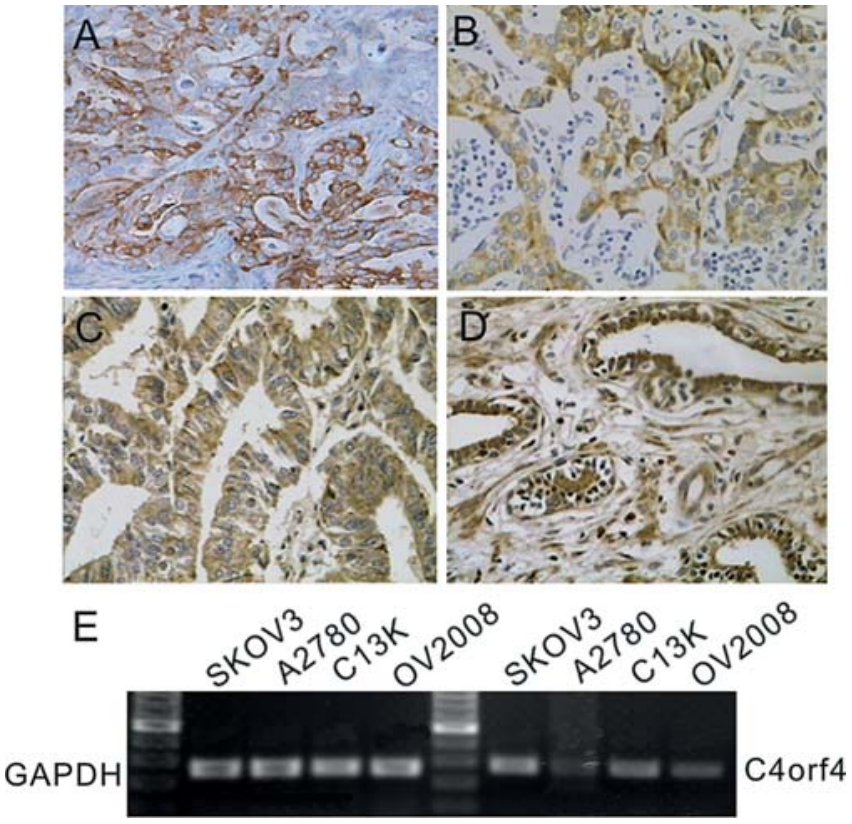

Figure 1. Expression and distribution of C4orf7 in tumor tissues and cell lines. Immunohistochemical staining for $\mathrm{C} 4$ orf7 was done as described in Materials and methods on paraffin-embedded, formalin-fixed tumor tissues, including epithelial ovarian carcinoma (A), breast cancer (B), endometrial carcinoma (C), prostate cancer (D). Original magnification, x200. (E) C4orf7 mRNA expression levels in A2780, SKOV3, C13K and OV2008 cell lines were determined by using RT-PCR.

cell motility was calculated as the wound width (at 0 and $24 \mathrm{~h})$. Values from at least three independent experiments for each sample were pooled and expressed as means \pm standard deviation (SD).

Migration and invasion assay. Cells stably expressing C4orf7 were resuspended at a density of $5.0 \times 10^{5} / \mathrm{ml}$ in serum-free medium. The cell suspension $(200 \mu \mathrm{l})$ was added to the upper chamber of an $8-\mu \mathrm{m}$ pore-size transwell inserts (Costar Corp., Cambridge, MA). The outer chambers were filled with $0.5 \mathrm{ml}$ of media containing $10 \%$ FBS. At $20 \mathrm{~h}$ after incubation at $37^{\circ} \mathrm{C}$, the cells were fixed with $4 \%$ paraformaldehyde in PBS and the non-migratory cells on the upper surface of the membrane were removed. The cells were stained in $0.1 \%$ crystal violet and images were captured (Olympus). The stained cells were washed and solubilized by using $10 \%$ acetic acid, then the absorbance was measured at $595 \mathrm{~nm}$. Values were normalized to empty pEGFP-C1 in each experiment.

Invasion assays were carried out in a similar manner to migration assays. Transwell inserts with $8-\mu \mathrm{m}$ pores (Costar Corp.) were coated with $35 \mu 1$ Matrigel, which was diluted 1:3 in ice-cold DMEM, and allowed to gel at $37^{\circ} \mathrm{C}$. Cells $\left(2.5 \times 10^{4}\right)$ in serum-free DMEM plated in the upper chamber. The cells were incubated for $30 \mathrm{~h}$ at $37^{\circ} \mathrm{C}$, and then fixed and stained as described above. All experiments were repeated in three replicates and experiments were repeated three times.

Cytoskeleton and cell immunofluorescence. Cells were plated overnight onto coverslips, washed three times with PBS
Table I. Correlation between expression of C4orf7 and clinicopathological features of epithelial ovarian carcinoma.

\begin{tabular}{lcccr}
\hline & & \multicolumn{2}{c}{ C4orf7 expression } & \\
\cline { 3 - 4 } Factor & No. of cases & Positive & Negative & P-value \\
\hline Age & & & & \\
$\quad<50$ & 49 & 31 & 18 & 0.859 \\
$\geq 50$ & 31 & 19 & 12 & \\
Grade & & & & \\
I and II & 60 & 36 & 24 & 0.424 \\
III & 20 & 14 & 6 & \\
Stage & & & & \\
I and II & 55 & 30 & 25 & 0.029 \\
III and IV & 25 & 20 & 5 & \\
LN metastasis & & & & \\
$\quad$ Negative & 41 & 18 & 23 & $<0.001$ \\
Positive & 39 & 32 & 7 & \\
\hline
\end{tabular}

${ }^{\mathrm{a}} \chi^{2}$ test; ${ }^{\mathrm{b}}$ Lymph node.

and fixed with $4 \%$ paraformaldehyde for $10 \mathrm{~min}$. Then cells were permeabilized with $0.1 \%$ Triton $\mathrm{X}-100$ for $15 \mathrm{~min}$ at $4^{\circ} \mathrm{C}$ and blocked with $1 \%$ BSA in PBS for $1 \mathrm{~h}$ at $37^{\circ} \mathrm{C}$. Rhodamine phalloidin (Eugene, OR) was used for staining of cytoskeleton (F-actin). The slides were observed by using a Leica TCS SP2 confocal microscope (Leica Microsystems, Ontario, Canada).

Statistical analysis. All experiments were repeated at least three times. SPSS 13.0 statistical software was used to perform statistical analysis. Students' t-test and $\chi^{2}$ test were used to evaluate the differences. The level of $\mathrm{P}<0.05$ was considered to be statistically significant.

\section{Results}

C4orf7 expression in tumor tissues. The expression of C4orf7 was tested by using immunohistochemical staining in epithelial ovarian carcinoma, breast cancer, endometrial carcinoma, and prostate cancer. As shown in Fig. 1, the expression of C4orf7 was positively detected in tumor cells of primary carcinoma tissues. The positive expression of C4orf7 was $70.0 \%$ in epithelial ovarian carcinoma, $61.35 \%$ in breast cancer, $67.4 \%$ in endometrial carcinoma, and $68.25 \%$ in prostate cancer. In the tissues of normal ovarian and border-line tumors, the C4orf7 expression was absent or rare. In order to determine the relationship between $\mathrm{C} 4$ orf7 expression and the clinical parameters of epithelial ovarian carcinoma or breast cancer, the basic characteristics of 80 epithelial ovarian carcinoma patients and 163 breast cancer patients were analyzed with respect to C4orf7 expression. As shown in Tables I and II, the percentages of C4orf7 positive expression in the tumor patients with metastases were significantly higher than those in the cancer without metastases, 
Table II. Correlation between expression of C4orf7 and clinicopathological features of breast carcinoma.

\begin{tabular}{lcccc}
\hline & & \multicolumn{2}{c}{ C4orf7 expression } & \\
\cline { 3 - 4 } Factor & No. of cases & Positive & Negative & P-value \\
\hline Age & & & & \\
$<50$ & 69 & 62 & 37 & 0.677 \\
$\geq 50$ & & 38 & 26 & \\
Grade & 123 & 71 & 52 & 0.095 \\
I and II & 40 & 29 & 11 & \\
III & & & & \\
Stage & 112 & 59 & 53 & 0.001 \\
I and II & 51 & 41 & 10 & \\
III and IV & & & & \\
LN & & & & \\
$\quad$ Negative & 84 & 29 & 55 & $<0.001$ \\
Positive & 79 & 71 & 8 & \\
\hline
\end{tabular}

${ }^{\mathrm{a}} \chi^{2}$ test; ${ }^{\mathrm{b}}$ Lymph node.

which suggested that C4orf7 expression was universal in human solid tumors and its immunoreactivity was strongly correlated with tumor metastasis.

Modulation of C4orf7 expression. We tested C4orf7 expression of ovarian cancer cell lines at mRNA level. As shown in Fig. 1E, among 4 cell lines, SKOV3 cells had the highest expression level and A2780 cells had the lowest expression level.

In order to study the effects of $\mathrm{C} 4$ orf7 protein, we prepared an expression vector encoding C4orf7 fused to a GFP epitope tag and transfected the construct into ovarian cancer cell line A2780. Another expression vector was also constructed with C4orf7 antisense RNA inserted, ovarian cancer cell line SKOV3 was transfected and stable cell lines developed. We determined expression of $\mathrm{C} 4$ orf7 by realtime-PCR. More than 2-fold overexpression of C4orf7 was detected in A2780/C4orf7 cells compared to vector control. In addition, Western blotting was used to detect the tag protein GFP. Together, the data above demonstrated that C4orf7 expression can be modulated by ectopic expression constructs in ovarian cancer cell lines, which can be used to investigate the biological behavior of this protein.

C4orf7 stimulates cell migration and invasion. To evaluate whether C4orf7 expression affects motility of ovarian cancer cell lines, a wound healing experiment was performed. The results shown in Fig. $2 \mathrm{C}$ demonstrated that $\mathrm{C} 4$ orf7 transfection increased the motility of A2780 cells and antisenseC4orf7 transfection delayed the motility of SKOV3 cells in a time-dependent manner compared to that of control cells Motility assay was performed in vitro by testing the cell motility from the top well to the lower chamber. The results were consistent with the wound healing assay in the same cell lines.

Furthermore, we assayed the capacity of A2780/C4orf7 cells to invade through matrigel-coated transwell inserts, as shown in Fig. 2, which was consistent with the results of migration assay. C4orf7 overpressed in A2780/C4orf7 cells tended to have enhanced in vitro invasion capacity.

The change of cytoskeleton. Cell migration is strictly regulated by re-organization of the actin cytoskeleton and focal adhesions (12). Previous studies revealed that tumor cells with different motile activities differ in terms of morphology, and that such differences might be attributable to the reorganization of the actin cytoskeleton, which generates intracellular tension (13-15). Immunofluorescence was used to examine the effects of C4orf7 expression on cell morpho$\operatorname{logy}$. To study how the changes of C4orf7 expression influence the reorganization of cytoskeleton, rhodaminephalloidin was used to label F-actin.

The cell shapes of the A2780/C4orf7 clones investigated showed polygonal cell shapes, in which brightly stained, longitudinal actin bundles were formed. F-actin was almost invisible at the cell edge (Fig. 3A and B). In contrast to vector control cells, we observed shrunken cell shapes in C4orf7 low-expressing clones, and F-actin polymerized at the edge of cells and stress fibers were almost invisible.

Enhancing C4orf7 expression leads to Akt ser473 phosphorylation and E-cadherin down-regulation. As shown in Fig. 3C, in our model cell line A2780, the overexpression of C4orf7 resulted in Akt ser473 phosphorylation. Akt ser473 phosphorylation is one of the activated forms of Akt, which plays important roles in tumorigenesis and tumor progression $(16,17)$. It is now generally accepted that E-cadherin is the main suppressor of epithelial tumor invasion. Decrease or disappearance of E-cadherin expression is described in many human carcinomas (18-21). We therefore, assayed the expression of E-cadherin, as shown in Fig. 3C, Western blot assays showed a decrease of E-cadherin expression in our model cell line A2780.

\section{Discussion}

Invasion and metastasis are distinguishing features of malignancy, and the main reasons for mortality. It is a complicated biological process involving numorous functional genes and a number of signal transduction pathways. More and more attention is paid to the research of this process, as new methods emerged and novel gene functions are detected. In our previous study, the results of gene microarray indicated that $\mathrm{C} 4$ orf7 has remarkable difference in expression level in breast carcinoma compared to normal tissues. The expression distributions of $\mathrm{C} 4$ orf7 was for the first time found to be expressed in FLT-4 positive LECs and cancer cells in breast cancer.

The goal of this study was to further investigate the function of this gene. C4orf7 lies on chromosome 4q13 adjacent to the cluster of C-X-C chemokines (4q chemokines) that includes IL-8 and other chemokines. While C4orf7 does not share significant primary sequence homolog 

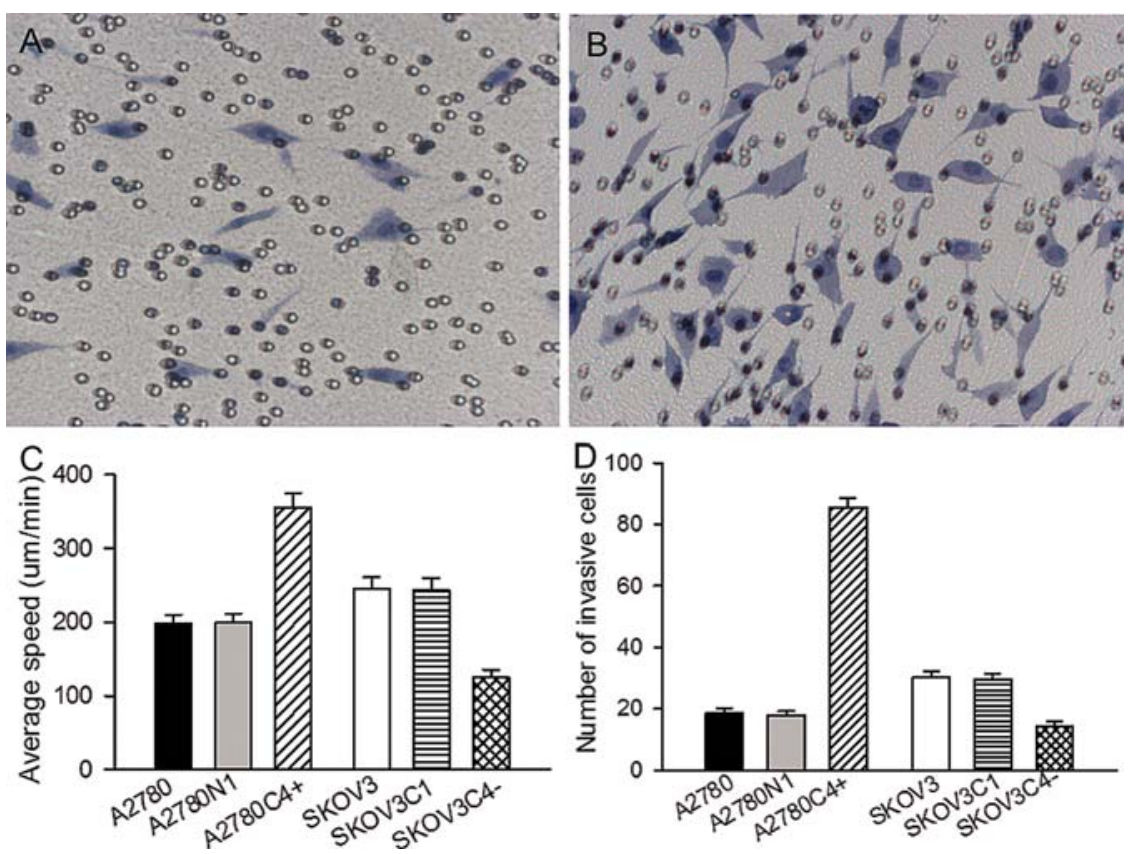

Figure 2. The effect of C4orf7 on motility and invasion. Cell invasion capability was detected by using transwell assay in enhanced pEGFPN1 A2780 cell group (A) and enhanced pEGFPN1-C4orf7 A2780 cell group (B). (C) Cell migration capability was detected by using wound healing assay in enhanced pEGFN1 or pEGFPN1-C4orf7 A2780 cells (A2780N1/A2780C4+) and pEGFPC1 or pEGFPC1-C4orf7 SKOV3 cells (SKOV3C1/SKOV3C4-). The distance of wound closure (compared with control at $0 \mathrm{~h}$ ) was measured in three independent wound sites per group. Values from at least three independent experiments were pooled and expressed as means \pm standard deviation (SD). (D) Invasion assay represented by numbers of cells passing through Matrigelcoated filters. Values represent means $\pm \mathrm{SE}$ from the three independent experiments.
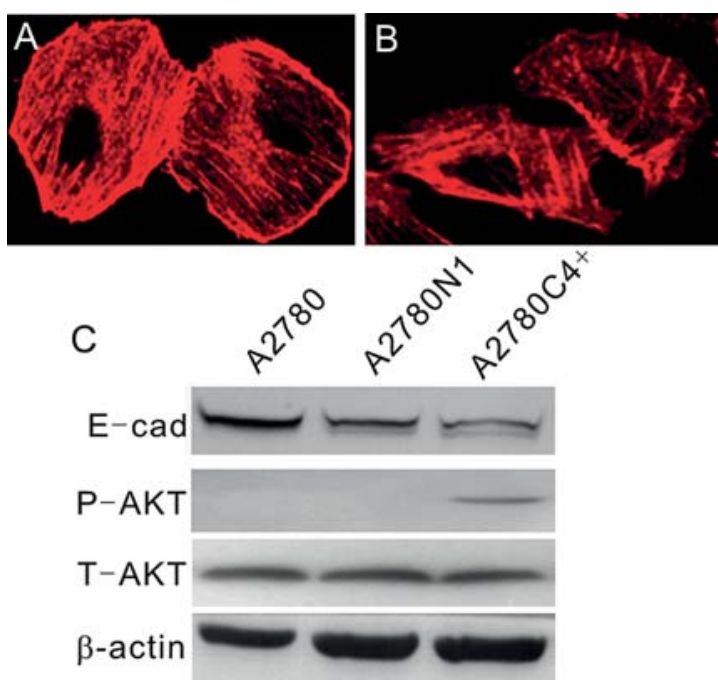

Figure 3. The effect of C4orf7 on cytoskeleton and phosphorylated Akt (p-AKT ${ }^{\text {Ser473) }}$ expression of A2780 cells in vitro. Cytoskeleton was stained with rhodamine-phalloidin and observed under a confocal laser scanning microscope. (A) Actin staining in untreated A2780 cells. (B) Actin staining in enhanced pEGFPN1-C4orf7 A2780 cells. (C) Western blot analysis of whole-cell lysates of A2780 cell lines stably expressing C4orf7 (A2780C4+) or pEGFPN1 (A2780N1). Cells were analyzed by using immuno-blotting with specific antibodies including E-cadherin (E-cad), phosphorylated Akt (P-AKT), total Akt (T-AKT), and B-actin. B-actin was used to demonstrate equal loading.

with chemokines, the overall amino acid composition, molecular mass, and charge (pI) of C4orf7 is similar to known inflammatory mediators such as IL-8 (9).
In order to validate gene microarray results, immunohistochemistry was carried out to detect C4orf7 expression in several tumor types. Here we reported the overexpression of gene C4orf7 in a variety of tumor types including breast carcinoma, epithelial ovarian carcinoma, and endometrial carcinoma. C4orf7 was expressed mainly in cancer cells and endothelial cells. In contrast, it was hardly expressed in corresponding normal tissues or benign lesions. It is reported that more than 30 sequences matching $\mathrm{C} 4$ orf7 are largely derived from fetal tissue or tumor cell libraries (9), which was consistent with our study. We supposed it may play roles in tumorigenesis or tumor metastasis.

Considering its localization and molecular features similar to CXC chemokines, we posed the question whether C4orf7 could influence tumor cell motility. It has been reported that during in vitro studies, C4orf7 promoted the migration of activated B lymphoma cells (10). We hypothesize that $\mathrm{C} 4$ orf7 functions in cell motility by binding target cells through a specific signaling receptor, analogous to chemokines. In our studies, wound healing assay and transwell assay were used to assess whether C4orf7 has effects on migration and invasion of ovarian cancer cells. In vitro, when human ovarian cancer cells A2780 and SKOV3 were exposed to $\mathrm{C} 4$ orf7 protein, they became more migratory. In addition, C4orf7 transfected A2780 cells migrated faster than vector control cells. Similarly, when C4orf7 was down-regulated by an antisense vector, there was an associated decrease in ovarian cancer cell migration and invasiveness. Collectively, these results indicated that $\mathrm{C} 4$ orf7 may play roles in ovarian cancer cell invasion and metastasis. 
Although the molecular mechanism by which C4orf7 has effects on ovarian cancer cell motility and invasiveness was not elucidated, we hypothesized that it may correlate with the alteration of cytoskeleton. The actin cytoskeleton is a highly dynamic network composed of actin polymers and a large variety of associated proteins $(22,23)$. In this study, Rhodamine phalloidin was used to label the F-actin cytoskeleton, and we observed C4orf7 up-regulated cells presented pseudopodia extension and assembling of stress fibers whereas down-regulated cells displayed a dramatic reorganization of actin network with actin polymerized at the edge of cells.

In the metastasis process, cancer cells should acquire their aggressive phenotype $(24,25)$. Motility of cancer cells plays a critical role in tumor metastasis, and as such is a target for intervention. Increasing evidence indicates that AKT activation is frequently in human epithelial cancers $(26,27)$. Especially in EOT (epithelial ovarian tumor), AKT2 activation was linked to aggressive clinical behavior and loss of histological features of epithelial differentiation (28). In this study, our data suggested that the high expression level of C4orf7 in ovarian cancer cells resulted in over-activation of Akt ser473 compared to control vectors.

As a result of up-regulation of the transcription repressor SNAIL, Akt activation represses E-cadherin gene transcription $(29,30)$. E-cadherin plays a central role in epithelial cell-cell adhesion and maintenance of epithelial cell colony integrity $(31,32)$. Loss or inhibition of the function of E-cadherin, or its associated proteins, $\beta$-catenin, results in reduced intercellular adhesions and acquisition of an invasive phenotype (33). Similarly, in our up-regulated C4orf7 cell lines, increased AKT phosphorylation (Ser473) and reduced levels of E-cadherin were observed as well as cell-cell adhesion decreased. The data may suggest that C4orf7 is indirectly associated with the E-cadherin/ß-catenin complex through regulating AKT Ser473 phosphorylation. Further work is now underway to determine the receptor of C4orf7 and to clarify the interactions of $\mathrm{C} 4$ orf7 with other cell-cell adhesion molecules.

In summary, we report the expression pattern of $\mathrm{C} 4$ orf7 in several tumor types. The expression level of C4orf7 was related to metastasis of epithelial ovarian cancer (EOC). In vitro, C4orf7 promotes EOC cells migration and invasiveness and decreases cell-cell adhension by phosphorylation of Akt s473 and down-regulation of E-cadherin. In addition, silencing of $\mathrm{C} 4$ orf7 caused cytoskeleton reorganization. Taken together, we demonstrated that $\mathrm{C} 4$ orf7 may play a role in progression of ovarian cancer, making it a candidate antineoplastic target.

\section{Acknowledgements}

Grants support: National Science Foundation of China (30973184; 30901586; 30528012) and the '973' Program of China (No. 2009CB521800).

\section{References}

1. Cannistra SA: Cancer of the ovary. N Engl J Med 351: 2519-2529, 2004.
2. Naora $\mathrm{H}$ and Montell DJ: Ovarian cancer metastasis: integrating insights from disparate model organisms. Nat Rev Cancer 5: 355-366, 2005 .

3. DiSaia PJ and Bloss JD: Treatment of ovarian cancer: new strategies. Gynecol Oncol 90: S24-S32, 2003.

4. Liu CM: Cancer of the ovary. N Engl J Med 352: 1268-1269, 2005.

5. Konstan PA, Spentzos D and Cannistra SA: Gene-expression profiling in epithelial ovarian cancer. Nat Clin Pract Oncol 5: 577-587, 2008.

6. Sundar SS and Ganesan TS: Role of lymphangiogenesis in cancer. J Clin Oncol 25: 298-307, 2007.

7. Karkkainen MJ, Makinen T and Alitalo K: Lymphatic endothelium: a new frontier of metastasis research. Nat Cell Biol 4: E2-E5, 2002.

8. Xiangyang B, Mei H, Jingyi Wu, et al: Development and characterization of a novel method to analyze global gene expression profiles in endothelial cells derived from primary tissues. Am J Hematol 83: 26-33, 2008.

9.Marshall AJ, Du Q, Draves KE, Shikishima Y, HayGlass KT and Clark EA: FDC-SP, a novel secreted protein expressed by follicular dendritic cells. J Immunol 169: 2381-2389, 2002.

10. Al-Alwan M, Du Q, Hou S, Nashed B, Fan Y, Yang X and Marshall AJ: Follicular dendritic cell secreted protein (FDC-SP) regulates germinal center and antibody responses. J Immunol 178: 7859-7867, 2007.

11. Li Q, Wu MF, Song AP, et al: Expression of ezrin and E-cadherin in invasive ductal breast cancer and their correlations to lymphatic metastasis. Ai Zheng (in Chinese) 25: 363-366, 2006.

12. Friedl $\mathrm{P}$ and Wolf $\mathrm{K}$ : Tumour-cell invasion and migration: diversity and escape mechanisms. Nat Rev Cancer 3: 362-374, 2003.

13. Nemethova M, Auinger S and Small J: Building the actin cytoskeleton: filopodia contribute to the construction of contractile bundles in the lamella. J Cell Biol 180: 1233-1244, 2008.

14. Hwang JH, Smith CA, Salhia B, et al: The role of fascin in the migration and invasiveness of malignant glioma cells. Neoplasia 10: 149-159, 2008.

15. Pollard TD and Borisy GG: Cellular motility driven by assembly and disassembly of actin filaments. Cell 112: 453-465, 2003.

16. Sun M, Wang G, Paciga JE, et al: AKT1/PKB kinase is frequently elevated in human cancers and its constitutive activation is required for oncogenic transformation in NIH3T3 cells. Am J Pathol 159: 431-437, 2001.

17. Arboleda MJ, Lyons JF, Kabbinavar FF, et al: Overexpression of $\mathrm{AKT} 2 /$ protein kinase Bbeta leads to up-regulation of beta1 integrins, increased invasion, and metastasis of human breast and ovarian cancer cells. Cancer Res 63: 196-206, 2003.

18. Yamaguchi H, Wyckoff J and Condeelis J: Cell migration in tumors. Curr Opin Cell Biol 17: 559-564, 2005.

19. Huber MA, Kraut N and Beug H: Molecular requirements for epithelial-mesenchymal transition during tumor progression. Curr Opin Cell Biol 17: 548-558, 2006.

20. Darai E, Scoazec JY, Walker-Combrouze F, et al: Expression of cadherins in benign, borderline, and malignant ovarian epithelial tumors: a clinicopathologic study of 60 cases. Hum Pathol 28: 922-928, 1997.

21. Sawada K, Mitra AK, Radjabi AR, et al: Loss of E-cadherin promotes ovarian cancer metastasis via alpha 5-integrin, which is a therapeutic target. Cancer Res 68: 2329-2339, 2008.

22. Valenzuela A and Cabrero JR: HDAC6: a key regulator of cytoskeleton, cell migration and cell-cell interactions. Trends Cell Biol 18: 291-297, 2008.

23. Bourguignon LY: Hyaluronan-mediated CD44 activation of RhoGTPase signaling and cytoskeleton function promotes tumor progression. Semin Cancer Biol 18: 251-259, 2008.

24. Wang W, Goswami S, Sahai E, et al: Tumor cells caught in the act of invading: their strategy for enhanced cell motility. Trends Cell Biol 15: 138-145, 2005.

25. Sahai E: Mechanisms of cancer cell invasion. Curr Opin Genet Dev 15: 87-96, 2005.

26. Larue L and Bellacosa A: Epithelial-mesenchymal transition in development and cancer: role of phosphatidylinositol 3' kinase/ AKT pathways. Oncogene 24: 7443-7454, 2005. 
27. Nicosia SV, Bai W and Cheng JQ: Oncogenic pathways implicated in ovarian epithelial cancer. Hematol Oncol Clin North Am 17: 927-943, 2003.

28. Tammela J and Odunsi K: Gene expression and prognostic significance in ovarian cancer. Minerva Gynecol 56: 495-502, 2004.

29. Grille SJ, Bellacosa A, Upson J, et al: The protein kinase akt induces epithelial mesenchymal transition and promotes enhanced motility and invasiveness of squamous cell carcinoma lines. Cancer Res 63: 21722-21728, 2003.

30. Blechschmidt K, Sassen S, Schmalfeldt B, et al: The Ecadherin repressor Snail is associated with lower overall survival of ovarian cancer patients. Br J Cancer 98: 489-495, 2008
31. Hudson LG, Zeineldin R and Stack MS: Phenotypic plasticity of neoplastic ovarian epithelium: unique cadherin profiles in tumor progression. Clin Exp Metastasis 25: 643-655, 2008.

32. Lee MY, Chou CY and Tang MJ: Epithelial-mesenchymal transition in cervical cancer: correlation with tumor progression, epidermal growth factor receptor overexpression, and snail upregulation. Clin Cancer Res 14: 4743-4750, 2008.

33. Thiery JP: Epithelial mesenchymal transitions in tumour progression. Nat Rev Cancer 2: 442-454, 2002. 